\title{
VIABILIDADE TÉCNICA E ECONÔMICA DA APLICAÇÃO DE ÁGUA NA CULTURA DO FEIJOEIRO COMUM (Phaseolus vulgaris L.)
}

\author{
Technical and economic viability of the application of water on the \\ culture of common bean (Phaseolus vulgaris L.)
}

\author{
Márcio José de Santana', Jacinto de Assunção Carvalho², Messias José Bastos de Andrade, \\ Gilson Gerson Gervásio ${ }^{4}$, José Carlos Braga ${ }^{5}$, Eduardo Branquinho Lepri ${ }^{6}$
}

\begin{abstract}
RESUMO
Este experimento foi conduzido no campus II do Centro Universitário de Patos de Minas (UNIPAM) em Patos de Minas, MG, durante o ano de 2005, e objetivou-se avaliar o rendimento do feijoeiro comum-cultivar Talismã submetida a diferentes níveis de reposição da água de irrigação, determinar a lâmina ótima econômica e a época de suspensão da irrigação para a cultura. Os tratamentos constaram de 5 níveis de reposição de água no solo $(40 \%, 70 \%, 100 \%, 130 \%$ e 160\%), em função da lâmina para elevá-lo diariamente à capacidade de campo e 3 épocas de suspensão da irrigação $(0,7$ e 14 dias após atingir o estádio R9 do ciclo cultural - modificação da cor das vagens, maturidade fisiológica das plantas). Utilizou-se para a irrigação um sistema por microaspersão. O delineamento experimental foi um DBC (blocos casualizados) em esquema fatorial de $5 \times 3 \mathrm{com} 4$ blocos. Foram avaliadas a produtividade da cultura e as lâminas ótima física e econômica. A produtividade da cultura foi afetada pelos níveis de reposição de água no solo (houve aumento da produtividade até reposição de $100 \%$ com posterior queda) e pelas épocas de suspensão da irrigação exceto na reposição de $100 \%$. As lâminas e produtividades que proporcionaram a máxima eficiência econômica foram 589,97 mm e 3205,76 kg ha ${ }^{-1}(\mathrm{R} 9), 535,72 \mathrm{~mm}$ e $2874,26 \mathrm{~kg} \mathrm{ha}^{-1}(\mathrm{R} 9+7)$ e $575,84 \mathrm{~mm}$ e $3242,73 \mathrm{~kg} \mathrm{ha}^{-1}(\mathrm{R} 9+14)$.
\end{abstract}

Termos para indexação: Manejo da irrigação, função de produção, lâmina ótima econômica.

\section{ABSTRACT}

This experiment was carried out at campus II of Patos de Minas University Center (Centro Universitário de Patos de Minas (UNIPAM) in Patos de Minas, MG, during the year of 2005, with the purpose of evaluating the yield of the common bean plant, cultivar Talismã, submitted to different levels of irrigation water replacement, as well as determining the optimum economic water height and the time to withhold the irrigation. The treatments consisted of five levels of water replacement in soil $(40 \%, 70 \%, 100 \%$, $130 \%$, and $160 \%)$ as a function of daily raising the height to the field capacity and three periods of irrigation withholding $(0,7$, and 14 days after reaching the $\mathrm{R} 9$ stage of the cropping cycle). A microsprinkling system was used for irrigation. The experimental design was a RBD (randomized blocks) with a factorial scheme of $5 \times 3$ with four blocks. The crop yield and the physical and economic optimum heights were evaluated. The crop yield was affected by the levels of water replacement in the soil (there was an increase of yield up to $100 \%$ replacement with further decrease) and by the time of irrigation withholding except in the replacement of $100 \%$. The water heights and yields, which afforded the maximum economic efficiency, were $589.97 \mathrm{~mm}$ and $3205.76 \mathrm{~kg} \mathrm{ha}^{-1}$ (R9), $535.72 \mathrm{~mm}$ and $2874.26 \mathrm{~kg} \mathrm{ha}^{-}(\mathrm{R} 9+7)$ and $575,84 \mathrm{~mm}$ and $3242.73 \mathrm{~kg} \mathrm{ha}^{-1}(\mathrm{R} 9+14)$

Index terms: Irrigation management, production function, optimum economic water height.

\section{(Recebido em 2 de fevereiro de 2007 e aprovado em 21 de junho de 2007)}

\section{INTRODUÇÃO}

O feijão representa importante fonte de diversos nutrientes e fibra, e sua composição pode variar de acordo com o local de plantio, fatores ambientais e com a cultivar. A área plantada na safra 2005/2006 foi de aproximadamente 4.200.000 hectares resultando em uma produção nacional de 3.448.000 toneladas do produto (CONAB, 2006). As baixas produção e produtividade têm sido atribuídas ao uso de cultivares pouco produtivas, aos efeitos desfavoráveis do solo (principalmente baixa fertilidade, pela expansão da cultura para áreas marginais), às condições adversas de clima e à incidência de pragas e doenças (GARRIDO, 1998).

\footnotetext{
'Engenheiro Agrônomo, Doutor, Professor - Setor de Olericultura - Centro Federal de Educação Tecnológica de Uberaba/CEFET - Rua João Batista Ribeiro, 4000 - Mercês - 38064-790 - Uberaba, MG - marciosantana@cefetuberaba edu.br - Bolsista CAPES

2Doutor em Engenharia Agrícola, Professor - Departamento de Engenharia/DEG - Universidade Federal de Lavras/UFLA - Cx. P. 3037 - $37200-000$ Lavras, MG - jacintoc@ufla.br

${ }^{3}$ Doutor, Professor - Departamento de Agricultura/DAG - Universidade Federal de Lavras/UFLA - Cx. P. 3037 - $37200-000$ - Lavras, MG mandrade@ufla.br

${ }^{4}$ Engenheiro Agrônomo - Rua Major Gote, 808 - Caiçaras - 38700-000 - Patos de Minas, MG - gilsonagro@yahoo.com.br

${ }^{5}$ Engenheiro Agrônomo - Rua Antônio Caetano de Menezes, 322 - Bela Vista - 38700-000 - Patos de Minas, MG - braga2@yahoo.com.br

${ }^{6}$ Engenheiro Agrônomo - Rua Afonso Queiroz - Fazenda Canavial - 38700-000 - Patos de Minas, MG - branquinholepri@yahoo.com.br
} 
Entre os tratos culturais, a irrigação constitui alternativa viável para substancial melhoria da produtividade. A finalidade básica da irrigação é proporcionar água à cultura de maneira a atender toda a exigência hídrica durante o ciclo cultural. Todavia, irrigações e sistemas mal dimensionados, com aplicações em excesso ou com déficit, poderão comprometer essa produtividade.

Faltam informações e pesquisas em diversos temas relacionados à relação água-solo-planta, em determinados locais. Devem ser realizados trabalhos que estudem alguns aspectos como a quantidade de água a ser aplicada de acordo com a cultivar e o solo, a resposta da cultura ao déficit e ao excesso de água e lâmina ótima econômica, entre outros. Esses estudos, preferencialmente, deverão estar ligados à realidade do produtor.

Outro problema enfrentado por produtores é a decisão sobre a época de suspender a irrigação do feijoeiro. Alguns irrigam até às vésperas da colheita, enquanto outros interrompem no início do estádio R9 (mudança da cor das vagens ou maturidade das vagens).

Dos fatores de produção, a água e os fertilizantes são aqueles que limitam os rendimentos com maior frequiência. Desse modo, o controle da irrigação e da fertilidade do solo constitui critério preponderante para o êxito da agricultura. A utilização das funções de produção permite encontrar soluções úteis na otimização do uso da água e dos fertilizantes na agricultura ou na previsão de rendimentos culturais (FRIZZONE, 1986).

Oliveira (1993) mencionou que muitos trabalhos de pesquisa envolvendo irrigação e fertilizantes apontam recomendações genéricas que objetivam a obtenção de produtividades físicas máximas, sem qualquer preocupação com a economicidade. A utilização da irrigação, com base nessas informações, poderá torná-la inviável do ponto de vista econômico, já que o ótimo econômico, geralmente, não corresponde à máxima produtividade biológica.

O presente trabalho foi planejado objetivando-se avaliar o desempenho do feijoeiro comum submetido às diferentes níveis de reposição de água no solo, determinar o efeito das lâminas de água nas produtividades física e econômica da cultura e avaliar a melhor época de suspensão da irrigação.

\section{MATERIAL E MÉTODOS}

O experimento foi conduzido em área experimental do Campus II do Centro Universitário de Patos de Minas (UNIPAM), no município de Patos de Minas, MG, localizado a $18^{\circ} 33^{\prime}$ '54' de latitude sul e $46^{\circ} 28^{\prime} 44^{\prime \prime}$ de longitude oeste, a uma altitude média de $835 \mathrm{~m}$, durante o período de julho a outubro de 2005. O solo da área experimental foi classificado como Latossolo Vermelho distroférrico.

A cultivar empregada foi a BRS-MG- Talismã. O experimento foi conduzido em blocos casualizados (DBC) com quatro repetições, sendo empregado um esquema fatorial $5 \times 3$, constituído por cinco níveis de reposição de água no solo $(40 \%, 70 \%, 100 \%, 130 \%$ e $160 \%$ da lâmina necessária para elevar a umidade do solo à capacidade de campo diariamente) e três épocas de suspensão da irrigação (0, 7 e 14 dias após R9, ou seja, R9, R9+7 e R9+14). Cada parcela experimental foi constituída por 5 linhas de plantio com $3 \mathrm{~m}$ de comprimento espaçadas de $0,45 \mathrm{~m}$ e com densidade de 12 plantas por metro. Como útil foi considerada uma área de $3 \mathrm{~m}^{2}$, sempre demarcada por um "gabarito" de madeira. Antes do preparo do solo procedeuse à aplicação de calcário com base no resultado de análise química do solo, tomando-se o valor de saturação por bases de $60 \%$.

Em cada parcela foram utilizados 4 microaspersores com sobreposição de 50\%, da marca DAN 2001, autocompensantes, com vazão de $28 \mathrm{~L} \mathrm{~h}^{-1}$. A uniformidade de aplicação de água foi de $98 \%$, obtida dias antes da semeadura. $\mathrm{O}$ bombeamento foi realizado com um conjunto motobomba de $1,5 \mathrm{cv}$, a partir de uma caixa com capacidade para 5.000 L.

Em três parcelas do tratamento $100 \%$ c.c - R9+14 foram instaladas baterias de tensiômetros com o objetivo de controle da lâmina de irrigação. Cada bateria consistiu de 2 tensiômetros, a 10 e $30 \mathrm{~cm}$ de profundidade. A capacidade de campo média do solo foi de $34,59 \%(10 \mathrm{kPa})$ e $29,31 \%(9 \mathrm{kPa})$, respectivamente, para as camadas de 0 $20 \mathrm{~cm}$ e $20-40 \mathrm{~cm}$. O cálculo do tempo de irrigação foi realizado com base nos sensores de 10 e $30 \mathrm{~cm}$.

Com as tensões observadas, foram calculadas as umidades correspondentes, a partir das curvas características a 10 e $30 \mathrm{~cm}$. De posse dessas umidades e daquela correspondente à capacidade de campo, e ainda, considerando a profundidade do sistema radicular estratificada em duas subcamadas $(0-20 \mathrm{~cm}$ e $20-40 \mathrm{~cm})$, foram calculadas as lâminas de reposição.

Após a colheita, os grãos foram pesados, tiveram seu grau de umidade medido, para correção do peso para umidade de $13 \%$. A produtividade foi submetida a uma análise de variância, sendo os efeitos dos tratamentos estudados por meio de análise de regressão ou do teste de média Scott-Knott, conforme o caso.

O modelo utilizado para ajuste da função de produção foi um polinômio do segundo grau, conforme Equação 1: 


$$
y=f(w)=a+b \cdot w+c w^{2}
$$

em que: $\mathrm{y}=$ produtividade $\left(\mathrm{kg} \mathrm{ha}^{-1}\right) ; \mathrm{w}=$ lâmina total de água aplicada (mm) e a, b e c = parâmetros da equação.

Para abordagem econômica da irrigação em um determinado sistema de produção, Peri et al. (1979), citados por Frizzone (1986), introduziram o conceito de lâmina ótima econômica, correspondente à máxima receita líquida obtida. O lucro foi expresso pela Equação 2.

$$
L(W)=P y . ~ Y-P w . ~ W-C
$$

em que: $\mathrm{L}(\mathrm{W})=$ lucro $\left(\mathrm{R} \$ \mathrm{ha}^{-1}\right) ; \mathrm{Py}=$ preço do feijão $(\mathrm{R} \$$ $\left.\mathrm{kg}^{-1}\right) ; \mathrm{Pw}=$ preço do fator água $\left(\mathrm{R} \$ \mathrm{~mm}^{-1} \mathrm{ha}^{-1}\right) ; \mathrm{W}=$ lâmina total de água aplicada $(\mathrm{mm}) ; \mathrm{Y}=$ produtividade do feijão $\left(\mathrm{kg} \mathrm{ha}^{-1}\right)$ e $\mathrm{C}=$ custo dos fatores mantidos constantes no experimento $\left(\mathrm{R} \$ \mathrm{ha}^{-1}\right)$.

Py representou o preço médio $\left(\mathrm{R} \$ 1,40 \mathrm{~kg}^{-1}\right)$ do feijão no mês de outubro de 2006, no comércio do Alto Paranaíba, microrregião do estado de Minas Gerais, onde localiza-se o município de Patos de Minas, MG. Pw representa o custo do volume unitário de água, acrescido do custo de energia utilizada no bombeamento, da depreciação do sistema de irrigação e dos custos de operação e manutenção desse sistema, conforme menciona Pereira (2005). Para a composição do custo da irrigação a ser empregado na análise econômica, tomouse por base o sistema de pivô central típico da região com 60,01 ha. O custo desse sistema de irrigação foi de $\mathrm{R} \$ 250.000,00$ sendo o custo por hectare de $\mathrm{R} \$ 4.165,97$. O valor residual foi estimado em $20 \%$ do valor da aquisição, segundo Pereira (2005), o que equivale a R\$ $50.000,00$.

Considerando que o equipamento será utilizado por dois ciclos em cada ano e a vida útil é de 10 anos, a depreciação foi de $\mathrm{R} \$ 166,63$ por hectare, por ciclo (Equação 3). A manutenção e operação do sistema equivalem a $2 \%$ do valor de aquisição, o que representa $\mathrm{R} \$ 41,65$ por hectare e ciclo de produção.

$$
D p=\frac{(V c-V r)}{V u}
$$

em que:

$\mathrm{Dp}=$ depreciação do sistema $(\mathrm{R} \$) ; \mathrm{Vc}=$ valor de compra do sistema $(\mathrm{R} \$)$; $\mathrm{Vr}=$ valor residual do sistema $(\mathrm{R} \$)$ $\mathrm{e} \mathrm{Vu}=$ vida útil do sistema (anos).

O custo da energia para bombeamento varia de acordo com a classe de consumidor e o tipo de contrato. Considerou-se no presente trabalho, a classe consumo rural e o grupo de tensão A, em função do motor utilizado pelo sistema de irrigação (rede trifásica de 380 V). Foram considerados a demanda de potência $(\mathrm{kW})$ e o consumo de energia (kWh); adotou-se para a análise a Tarifa HoroSazonal, considerando Horário Fora de Ponta (HFP) e o Horário Fora de Ponta /Noturno (HFP/N), cujo valor representa $22 \%$ do valor cobrado pelo HFP (todos no período seco). O consumo de energia, a demanda de potência e o custo final de energia (já acrescidos os valores de impostos - 0,82) em $\mathrm{R} \$$ foram calculados com as Equações 4, 5 e 6.

$$
\begin{aligned}
& \text { Consumo }=2,64+0,8 . \text { Potência }(c v) \\
& \text { Demanda }=\frac{\text { Potência }(c v) \cdot 0,736 \mathrm{~kW} / \mathrm{cv}}{\operatorname{Re} n \operatorname{dim} \text { ento }(\text { decimal })}
\end{aligned}
$$

CustoFinal $=\frac{\left(D_{c} \cdot T_{d}\right)+\left(C_{f p} \cdot T_{f p} \cdot H_{f p}\right)+\left(C_{f p n} \cdot T_{f p n} \cdot H_{f p n}\right)}{0,82}$

em que: $D_{c}=$ demanda total contratada, $\mathrm{kW} ; T_{d}=$ tarifa de demanda, $\mathrm{R} \$ \mathrm{~kW}^{-1} ; C_{f p}=$ consumo de energia em horário fora de ponta, $\mathrm{kWh} ; T_{f p}=$ tarifa de consumo em horário fora de ponta, $\mathrm{R} \$ \mathrm{kWh}^{-1} ; H_{f p}=$ horas utilizadas no horário fora de ponta, $\mathrm{h} ; C_{f p n}=$ consumo de energia em horário fora de ponta/noturno, $\mathrm{kWh} ; T_{f p n}=$ tarifa de consumo em horário fora de ponta/noturno, $\mathrm{R} \$ \mathrm{kWh}^{-1}$ e $H_{f p n}=$ horas utilizadas no horário fora de ponta/ noturno, h.

Para cálculo da energia de bombeamento utilizou-se a lâmina aplicada no tratamento $100 \%$ c.c. - R9+14. Considerouse o pivô a $60 \%$ de velocidade no percentímetro, a cada irrigação com um total de 16,33 horas, sendo 6 horas de irrigação no HFP/N e 10,33 horas no HFP. A potência no eixo calculada foi de $92,68 \mathrm{cv}$ e a comercial de $100 \mathrm{cv}$, com rendimentos de motor e bomba de 0,9 e 0,73 , respectivamente. Os valores de tarifação para outubro de 2006 foram: demanda HFP de R\$10,2976 e consumo HFP período seco R $\$ 0,24288 \mathrm{~kW} \mathrm{~h}^{-1}$.

Substituindo todos os valores citados anteriormente nas Equações 4, 5 e 6, tem-se um custo de bombeamento de $\mathrm{R} \$ 308,63$ por hectare e ciclo de cultivo.

Não foi considerado o custo para o volume de água utilizado, sendo a captação considerada pública ou de uso do produtor. O custo do fator água (energia + manutenção) foi de $\mathrm{R} \$ 0,5456 \mathrm{~mm}^{-1} \mathrm{ha}^{-1}$.

Considerando que o lucro é máximo quando a primeira derivada do rendimento em relação à lâmina total de água for igual à relação entre fator e produto, tem-se: 


$$
\frac{\partial Y}{\partial W}=b+2 c W=\frac{P w}{P y}
$$

A lâmina ótima econômica foi obtida da Equação 7. Para obtenção do custo de produção do feijão foram tomadas informações contidas em Agrianual (2006), referentes a julho de 2005, com atualização dos preços para outubro de 2006, obtendo-se um valor de $\mathrm{R} \$ 2.920,78 \mathrm{ha}^{-1}$.

\section{RESULTADOS E DISCUSSÃO}

A análise de variância revelou efeito significativo das lâminas de reposição, das épocas de suspensão e da interação entre esses fatores (Tabela 1). Felipe (1991), Frizzone (1986) e Garrido (1998), também encontraram diferença significativa entre lâminas de irrigação aplicadas na cultura do feijoeiro.

Nas Figuras 1, 2 e 3 observa-se o comportamento da produtividade em $\mathrm{kg} \mathrm{ha}^{-1}$, em função do nível de reposição de água no solo, com emprego de diferentes épocas de suspensão da irrigação: R9, R9 + 7 e R9 + 14 dias, respectivamente.

Tabela 1 - Resumo da análise de variância dos dados relativos à produtividade do feijoeiro $\left(\mathrm{kg} \mathrm{ha}^{-1}\right)$. Patos de Minas, MG, 2005.

\begin{tabular}{lcc}
\hline \multicolumn{1}{c}{ Fontes de variação } & $\begin{array}{c}\text { Graus de } \\
\text { liberdade }\end{array}$ & $\begin{array}{c}\text { Quadrado } \\
\text { médio }\end{array}$ \\
\hline Reposição de água $(\mathrm{R})$ & 4 & $397442,7^{* *}$ \\
$\begin{array}{l}\text { Época suspensão } \\
\text { irrigação (E) }\end{array}$ & 2 & $473119,1^{* *}$ \\
$\mathrm{R} x \mathrm{E}$ & 8 & $335010,2^{* *}$ \\
Bloco & 3 & \\
Erro & 42 & \\
\hline $\mathrm{CV}(\%):$ & 10,30 & \\
\hline Média geral $\left(\mathrm{kg} \mathrm{ha}^{-1}\right):$ & 2.818 & \\
\hline
\end{tabular}

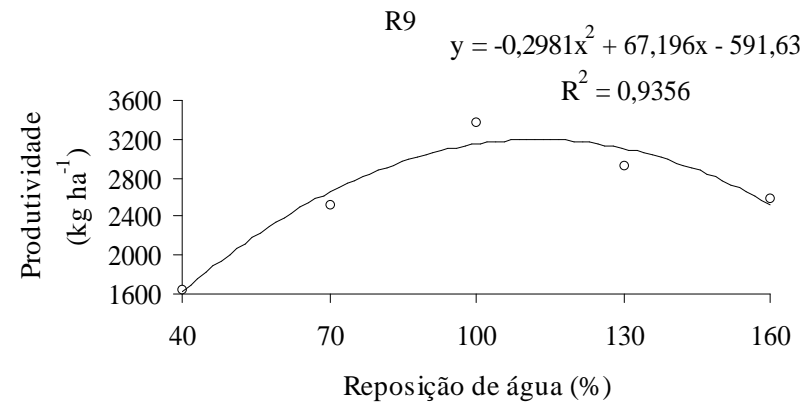

Figura 1 - Produtividade média em função do nível de reposição de água.

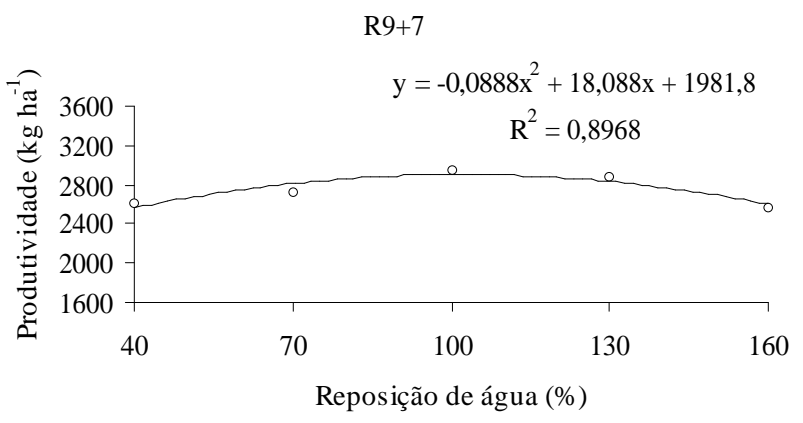

Figura 2 - Produtividade média em função do nível de reposição de água.

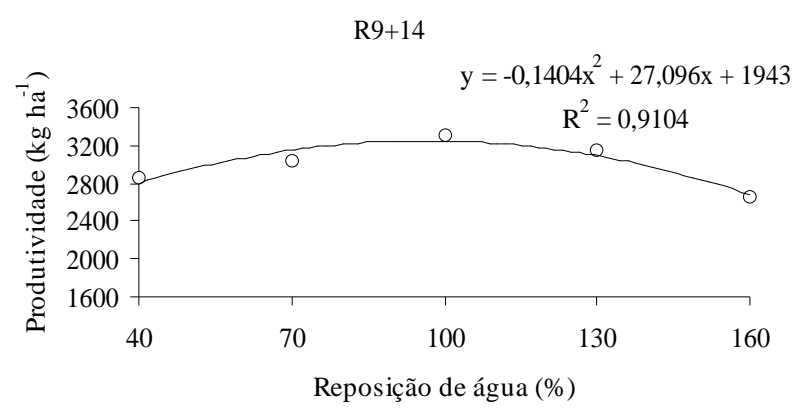

Figura 3 - Produtividade média em função do nível de reposição de água.

Verifica-se que, independente da época de suspensão da irrigação, há um aumento da produção em função do nível de reposição de água, atingindo um máximo com uma reposição de água correspondente a $100 \%$ de água consumida, havendo uma queda de produtividade após esse valor. Dessa forma, lâminas menores ou maiores ocasionaram perdas de produtividade. O déficit hídrico ocasiona murchamento das plantas, prejudicando sua fisiologia, culminando em queda de produtividade. O feijão é uma planta muito sensível ao estresse hídrico, por causa da sua baixa capacidade de recuperação após o déficit e ao seu sistema radicular pouco desenvolvido (GUIMARÃES, 1988). Por outro lado, lâminas excessivas provocam um ambiente com falta de aeração para as plantas, bem como favorece a disseminação de doenças e perdas de nutrientes por lixiviação. O feijoeiro é uma planta relativamente sensível ao excesso de água no solo, pois, nessa condição, o desenvolvimento vegetativo e o rendimento são prejudicados, pelo aumento da resistência do movimento da água através das raízes, menor absorção de nutrientes, menor aeração e aumento da concentração de substâncias tóxicas nas plantas (SILVEIRA \& STONE, 2001). A redução do rendimento da cultura com reposições excessivas também foi verificada por Frizzone (1986) e Garrido (1998). 
Os dados médios de produtividade para cada lâmina de reposição, dentro das diferentes épocas de suspensão da irrigação, observam-se na Tabela 2. A suspensão da irrigação em R9 resultou em menor produtividade em três dos níveis de reposição utilizados, mas na lâmina de $100 \%$ de reposição (a melhor delas) não houve diferenças significativas entre as épocas de suspensão da irrigação, indicando que, em termos de produtividade física, não se justifica continuar com a irrigação até muito próximo da colheita.

Os produtos físicos marginais (PFMa) foram obtidos a partir das funções de produção (Figuras 4, 5 e 6) estimadas para cada fase de suspensão da irrigação.

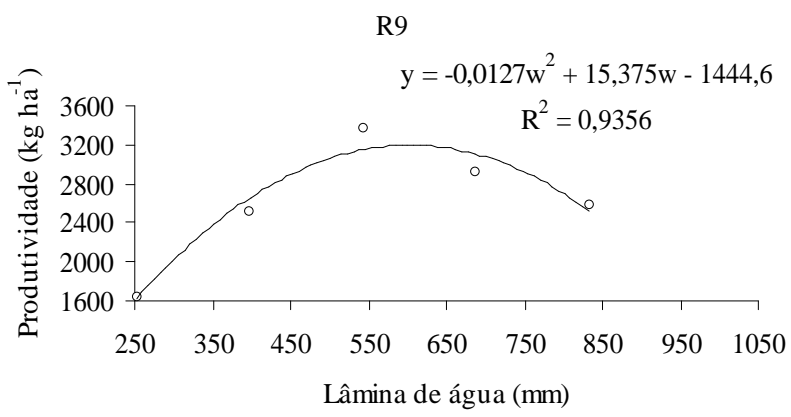

Figura 4 - Produtividade do feijoeiro cultivar Talismã, em função das lâminas de água.

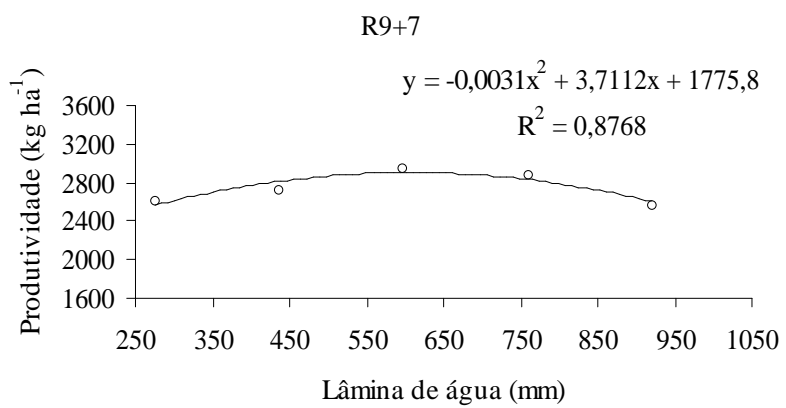

Figura 5 - Produtividade do feijoeiro cultivar Talismã, em função das lâminas de água.

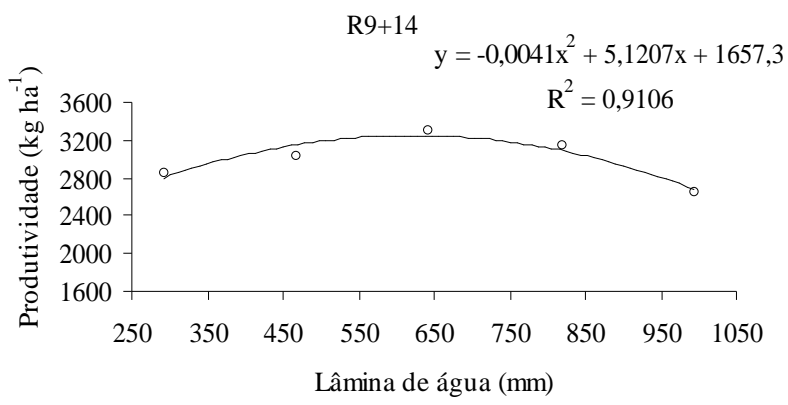

Figura 6 - Produtividade do feijoeiro cultivar Talismã, em função das lâminas de água.

Considerou-se como lâmina de água economicamente ótima aquela que conduziu ao rendimento com máxima margem bruta. Dessa forma derivou-se a estimativa das funções de produção, obtendo-se o valor do produto físico marginal (PFMa) e em seguida igualou-se esse à relação entre o preço do produto e o preço do fator água (PEREIRA, 2005).

$$
\begin{aligned}
& \frac{\partial Y}{\partial W}=15,375-0,0254 W=\frac{P w}{P y} \\
& \frac{\partial Y}{\partial W}=3,7112-0,0062 W=\frac{P w}{P y} \mathrm{e} \\
& \frac{\partial Y}{\partial W}=5,1166-0,084 W=\frac{P w}{P y}
\end{aligned}
$$

Quando o valor do PFMa atinge o valor zero concluise que a lâmina aplicada proporcionou a produtividade física máxima. Igualando-se a primeira derivada a zero $(\mathrm{PFMa}=0)$ as maiores produtividades físicas apresentamse na Tabela 3, para as épocas de suspensão da irrigação R9, R9+7 e R9+14. Frizonne (1986) trabalhando com a cultivar Carioca e seis lâminas de irrigação variando entre 105 e $621 \mathrm{~mm}$, observou uma resposta quadrática do rendimento da cultura, sendo a produtividade máxima de $1.871 \mathrm{~kg} \mathrm{ha}^{-1}$, para uma lâmina de $533 \mathrm{~mm}$. Azevedo (1984) constatou produtividade física máxima de $905 \mathrm{~kg} \mathrm{ha}^{-1} \mathrm{e}$

Tabela 2 - Produtividade média $\left(\mathrm{kg} \mathrm{ha}^{-1}\right)$ do feijoeiro comum para as diferentes reposições de água, em função das épocas de suspensão da irrigação.

\begin{tabular}{cccccc}
\hline \multirow{2}{*}{ Épocas } & \multicolumn{5}{c}{ Reposição água (\%) } \\
\cline { 2 - 6 } & 40 & 70 & 100 & 130 & 160 \\
\hline R9 & $1645,8 \mathrm{~b}^{1}$ & $2521,2 \mathrm{~b}$ & $3377,4 \mathrm{a}$ & $2928,0 \mathrm{~b}$ & $2578,0 \mathrm{a}$ \\
R9 +7 & $2601,0 \mathrm{a}$ & $2724,6 \mathrm{a}$ & $2940,0 \mathrm{a}$ & $2870,4 \mathrm{~b}$ & $2577,0 \mathrm{a}$ \\
R9 +14 & $2853,0 \mathrm{a}$ & $3033,6 \mathrm{a}$ & $3297,6 \mathrm{a}$ & $3144,6 \mathrm{a}$ & $2649,0 \mathrm{a}$ \\
\hline
\end{tabular}

${ }^{1}$ As médias seguidas pela mesma letra na vertical não diferem entre si, em 5\% de probabilidade, pelo teste Scott-Knott. 
lâmina de 394 mm, e Felipe (1991) de $1.436 \mathrm{~kg} \mathrm{ha}^{-1}$ e lâmina de $312 \mathrm{~mm}$. Os rendimentos máximos obtidos no atual experimento, bem como as correspondentes lâminas, foram superiores aos valores apresentados pelos autores citados.

Tabela 3 - Lâminas que proporcionaram máxima produtividade física (L.F.), produtividade física máxima (P.F.), lâminas que proporcionaram máxima produtividade econômica (L.O.), produtividade economicamente ótima (P.O.) e lucro, encontrados para as diferentes épocas de paralisação da irrigação.

\begin{tabular}{cccccl}
\hline Épocas & $\begin{array}{c}\text { L.F. } \\
(\mathrm{mm})\end{array}$ & $\begin{array}{c}\text { P.F. } \\
\left(\mathrm{kg} \mathrm{ha}^{-1}\right)\end{array}$ & $\begin{array}{c}\text { L.O. } \\
(\mathrm{mm})\end{array}$ & $\begin{array}{c}\text { P.O. } \\
\left(\mathrm{kg} \mathrm{ha}^{-1}\right)\end{array}$ & $\begin{array}{c}\text { Lucro } \\
\left(\mathrm{R} \$ \mathrm{ha}^{-1}\right)\end{array}$ \\
\hline R9 & 605,31 & $3.208,75$ & 589,97 & $3.205,76$ & $1.245,35$ \\
$\mathrm{R} 9+7$ & 598,58 & $2.886,52$ & 535,72 & $2.874,26$ & 810,89 \\
$\mathrm{R} 9+14$ & 623,97 & $3.251,99$ & 575,84 & $3.242,73$ & $1.304,86$ \\
\hline
\end{tabular}

Ainda na Tabela 3 são apresentadas as lâminas ótimas econômicas obtidas igualando-se as expressões dos valores de PFMa ao preço do fator variável. Ressaltase que, consideram-se os custos relativos ao mês de outubro de 2006, os lucros máximos para as épocas R9, R9+7 e R9+14, foram de R\$ 1.245,35; R \$ 810,89 e R\$ 1.304,86, respectivamente.

Na Figura 7 nota-se a variação da lâmina ótima econômica, em função da variação da relação $\mathrm{Pw} / \mathrm{Py}$, para as diferentes épocas de suspensão da irrigação. Os menores valores de lâminas economicamente ótimas ocorreram para $\mathrm{R} 9+7$, para uma mesma relação. Como exemplo analisa-se uma relação de $50 \%(\mathrm{Pw} / \mathrm{Py}=0,5)$, em que a lâmina ótima econômica foi de 585,62 mm, $517,93 \mathrm{~mm}$ e $549,59 \mathrm{~mm}$ para R9, R9+7 e R9+14, respectivamente.

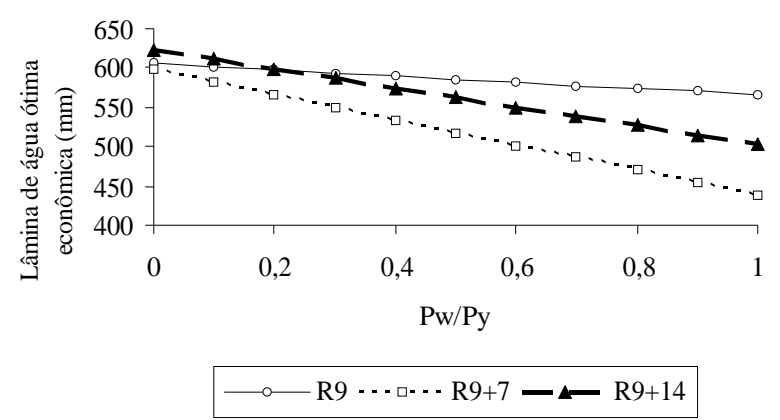

Figura 7 - Lâmina total de água economicamente ótima em função da relação entre o preço da água e o preço do feijão.

\section{CONCLUSÕES}

A cultura do feijoeiro cultivar Talismã quando submetida a lâminas deficitárias, bem como excessivas, reduziu negativamente sua produtividade. Os níveis de reposição afetaram as produtividades economicamente ótimas, sendo o maior valor quando irriga-se até R9+14 $\left(3.242,73 \mathrm{~kg} \mathrm{ha}^{-1}\right)$. As médias de produtividade observadas quando se irriga com $100 \%$ de reposição e suspensão em R9, R9+7 e R9+14, não foram estatisticamente diferentes.

\section{REFERÊNCIAS BIBLIOGRÁFICAS}

AGRIANUAL. Anuário da agricultura brasileira. São Paulo: FNP Consultoria e Comércio, 2006. 520 p.

AZEVEDO, H. J. Efeito de diferentes lâminas de água e doses de adubação nitrogenada na cultura do feijoeiro (Phaseolus vulgaris L.). 1984. 85 f. Dissertação (Mestrado em Irrigação) - Escola Superior de Agricultura “Luiz de Queiroz”, Piracicaba, 1984.

\section{COMPANHIA NACIONAL DEABASTECIMENTO.}

Histórico safras de grãos. Brasília, DF, 2006.

Disponível em: <http://Www.conab.gov 20 out. 2006.

FELIPE, M. P. Efeitos de diferentes lâminas de água e épocas de parcelamento da adubação nitrogenada na cultura do feijão (Phaseolus vulgaris L.). 1991. 105 f. Dissertação (Mestrado em Fitotecnia) - Escola Superior de Agricultura de Lavras, Lavras, 1991.

FRIZZONE, J. A. Funções de resposta do feijoeiro (Phaseolus vulgaris L.) ao uso de nitrogênio e lâminas de irrigação. 1986. 133 f. Tese (Doutorado em solos e nutrição de plantas) - Escola Superior de Agricultura "Luiz de Queiroz", Piracicaba, 1986.

GARRIDO, M. A. T. Respostas do feijoeiro às lâminas de água e adubação nitrogenada. 1998. 205 p. Tese (Doutorado em Fitotecnia) - Universidade Federal de Lavras, Lavras, 1998.

GUIMARÃES, C. M. Efeitos fisiológicos do estresse hídrico. In: ZIMMERMANN, M. J. O.; ROCHA, M.; YAMADA, T. Cultura do feijoeiro: fatores que afetam a produtividade. Piracicaba: Associação Brasileira para Pesquisa da Potassa e do Fosfato, 1988. p. 157-174. 
OLIVEIRA, S. L. Função de resposta do milho doce ao uso de irrigação e nitrogênio. 1993. 91 f. Tese

(Doutorado em Engenharia Agrícola) - Universidade Federal de Viçosa, Viçosa, 1993.

PEREIRA, J. R. D. Viabilidade técnica e econômica das aplicações de água e nitrogênio no cultivo de gladíolo
(Gladiolus $\boldsymbol{x}$ grandiflorus L.). 2005. 80 p. Tese

(Doutorado em Engenharia Agrícola) - Universidade

Federal de Lavras, Lavras, 2005.

SILVEIRA, P. M.; STONE, L. F. Irrigação do feijoeiro. Goiânia: Embrapa/CNPAF, 2001. 230 p. 